\title{
Protokoll der Mitgliederversammlung des Vereins Ultraschallmuseum e.V. am 27.10.2016 in Leipzig
}

Der Tagesordnung wird durch die MV zugestimmt.

1. Das Protokoll der MV DLT Davos vom 24.9.2015 wird bestätigt.

2. Bericht des Vorsitzenden:

- Die Gesellschaft für angewandte Ultraschallphysik GEFAU löst sich auf und hat dem Museum ihren großen
Bestand an Büchern und Zeitschriften angeboten. Da die DEGUM dieses Projekt mit $5000 €$ unterstützt, haben wir in Berlin im Lager etwa die Hälfte des Bestandes, ca. 50 Umzugskartons nach Lennep transportiert und dort in unser digitales $\mathrm{Ar}$ chiv unserer Homepage eingegeben und in 2 neue Regalsysteme eingeordnet. Damit verfügen wir über eine fast komplette Reihe von Zeitschriften und Bücher über die Ultraschallphysik.

- Gleichzeitig haben wir das DEGUM Archiv von Prof Rettenmaier übernommen. 
- In Linz werden wir ausschließlich unsere Kretz-Reihe präsentieren mit live Vorführungen und entsprechenden Postern.

- Wir haben beschlossen, die Ultraschallgeschichte aus deutscher Sicht zu schreiben: u. a. Geschichte der DAUD, DEGUM, DDR, EFSUMB u. a. und einige Pioniere zu würdigen. Unklar ist noch die Herausgabe und die Kostenübernahme.
- Gleichzeitig laufen Gespräche mit der EFSUMB über die Geschichte der EFSUMB - Mitgliedstaaten.

- Ein Gespräch mit Herrn Busch, dem Direktor des Röntgenmuseums hat gezeigt, dass er uns wohl gesonnen ist und die Verzögerung der Wiedereröffnung unseres Schaufensters nur an mangelnden Geldern liegt.

3. Der Finanzbericht zeigt ein Guthaben von $6128,53 €$
4. Der Erhöhung des Mitgliederbeitrages von 15 auf $25 €$ wird einstimmig zugestimmt

5. Auf Antrag von Herrn Lange wird der Vorstand für das Jahr 2015 einstimmig entlastet.

6. Der Vorstand hat sich noch einmal zur Wahl gestellt und wurde einstimmig gewählt: Vorsitzender: B. Frentzel-Beyme, stellverstretender Vorsitzender: C. Jakobeit, weiter H. Lutz, M. Salaschek und D. Nürnberg.

Protokoll: B. Frentzel-Beyme 